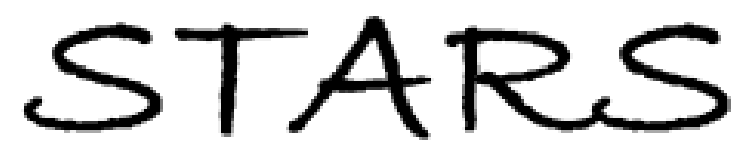

University of Central Florida

STARS

$1-1-2011$

\title{
Observation of the Plateau-Rayleigh capillary instability in multi- material optical fibers
}

\author{
S. Shabahang \\ University of Central Florida \\ J. J. Kaufman \\ University of Central Florida \\ D. S. Deng \\ A. F. Abouraddy \\ University of Central Florida
}

Find similar works at: https://stars.library.ucf.edu/facultybib2010

University of Central Florida Libraries http://library.ucf.edu

This Article is brought to you for free and open access by the Faculty Bibliography at STARS. It has been accepted for inclusion in Faculty Bibliography 2010 s by an authorized administrator of STARS. For more information, please contact STARS@ucf.edu.

\section{Recommended Citation}

Shabahang, S.; Kaufman, J. J.; Deng, D. S.; and Abouraddy, A. F., "Observation of the Plateau-Rayleigh capillary instability in multi-material optical fibers" (2011). Faculty Bibliography 2010s. 1886.

https://stars.library.ucf.edu/facultybib2010/1886

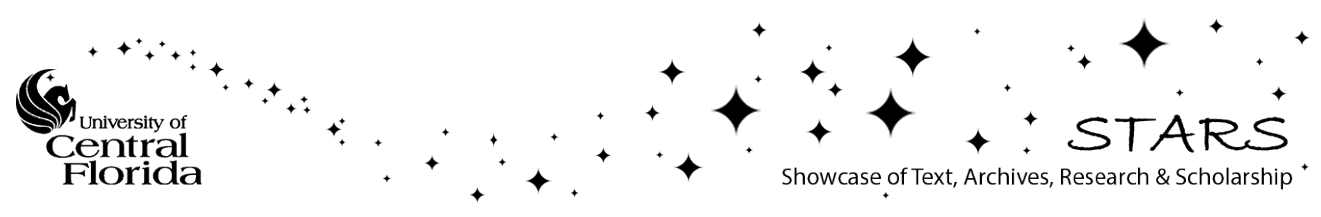




\section{Observation of the Plateau-Rayleigh capillary instability in multi-material optical fibers}

Cite as: Appl. Phys. Lett. 99, 161909 (2011); https://doi.org/10.1063/1.3653247

Submitted: 24 June 2011 . Accepted: 28 September 2011 . Published Online: 21 October 2011

S. Shabahang, J. J. Kaufman, D. S. Deng, and A. F. Abouraddy

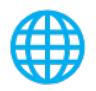

\section{ARTICLES YOU MAY BE INTERESTED IN}

On the breakup of viscous liquid threads

Physics of Fluids 7, 1529 (1995); https://doi.org/10.1063/1.868540

Slow growth of the Rayleigh-Plateau instability in aqueous two phase systems

Biomicrofluidics 6, 022007 (2012); https://doi.org/10.1063/1.3700117

The Rayleigh-Plateau instability and jet formation during the extrusion of liquid metal from craters in a vacuum arc cathode spot

Journal of Applied Physics 117, 043302 (2015); https://doi.org/10.1063/1.4906559

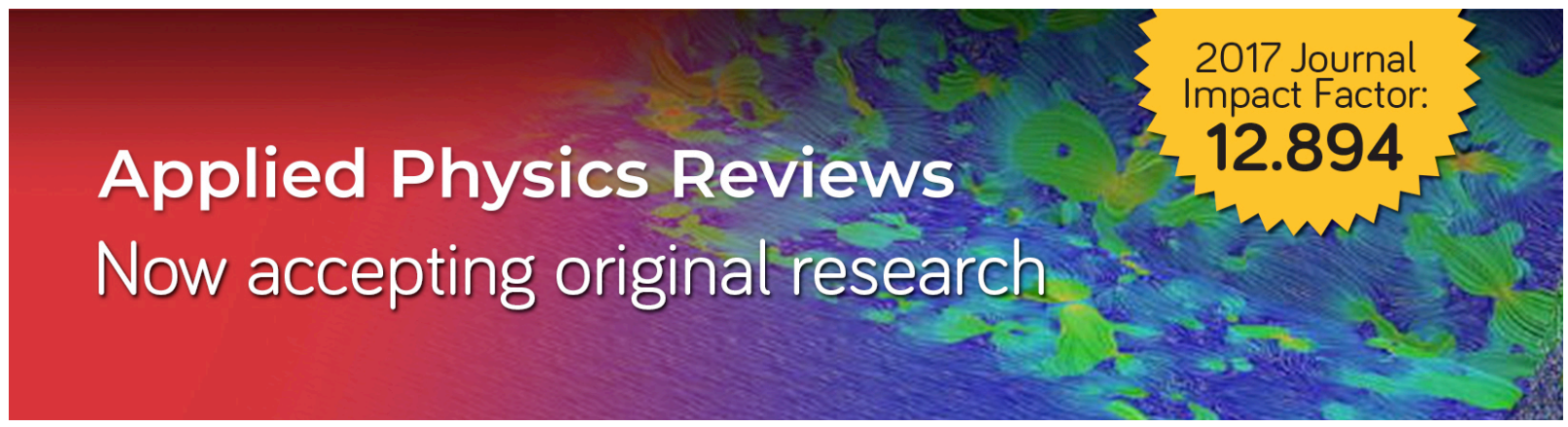




\title{
Observation of the Plateau-Rayleigh capillary instability in multi-material optical fibers
}

\author{
S. Shabahang, ${ }^{1}$ J. J. Kaufman, ${ }^{1}$ D. S. Deng, ${ }^{2}$ and A. F. Abouraddy ${ }^{1, a)}$ \\ ${ }^{1}$ CREOL, The College of Optics and Photonics, University of Central Florida, 4000 Central Florida Blvd., \\ Orlando, Florida 32816, USA \\ ${ }^{2}$ Department of Chemical Engineering, Massachusetts Institute of Technology, 77 Massachusetts Avenue, \\ Cambridge, Massachusetts 02139, USA
}

(Received 24 June 2011; accepted 28 September 2011; published online 21 October 2011)

\begin{abstract}
We report the observation of the Plateau-Rayleigh capillary instability during the tapering of a multi-material optical fiber. The fiber core is a glass, and the cladding is an amorphous polymer. The instability is manifested in the breakup of the core into a periodic string of size-tunable micro-scale droplets embedded along the fiber axis. The particle diameters may be tuned in the 1-20 $\mu \mathrm{m}$ range through control of the tapering speed and temperature. Extending this approach to the fabrication of polymer and glass nanoparticles appears feasible. (C) 2011 American Institute of Physics. [doi:10.1063/1.3653247]
\end{abstract}

As a descending water jet accelerates, it narrows and ultimately breaks up into droplets, a common everyday observation captured by the Plateau-Rayleigh (PR) capillary instability. ${ }^{1-4}$ Two competing classes of force give rise to this instability: (1) surface tension that minimizes the surface area and disrupts the jet and (2) inertia, ${ }^{5}$ viscous dissipation forces, ${ }^{6}$ or visco-elastic forces ${ }^{7}$ that maintain it. Hydrodynamic instabilities have recently become particularly pertinent to optical fiber fabrication with the development of photonic crystal fibers containing air holes ${ }^{8}$ and multi-material fibers where distinct solid materials are co-drawn to form novel fiber sensors ${ }^{9,10}$ and actuators. ${ }^{11}$ In contrast to traditional optical fibers where the core and cladding are virtually the same material, these novel fibers involve heterogeneous interfaces that are susceptible to instability growth during processing at elevated temperatures and low viscosity. For example, it has been established that scattering from frozen surface capillary waves is the dominant source of optical transmission loss in photonic crystal fibers ${ }^{12,13}$ and that fluid instabilities limit the reduction in feature size in multi-material fibers. ${ }^{14,15}$ Surfacetension-driven instabilities, which dominate at large surfacecurvature, are consequently expected to determine the smallest transverse size of an axially continuous feature in a multimaterial fiber. We present here the first observation of the PR capillary instability in a multi-material fiber at the corecladding interface. We observe a wide gamut of dynamical fluid phenomena in the fibers such as droplet pinch-off, growth of periodic droplet arrays, and inter-droplet satellite and sub-satellite formation. We also develop a quasi-static extension of Tomotika's linear stability analysis ${ }^{16}$ as a theoretical framework for our findings.

We use fiber tapering ${ }^{17,18}$ to study the effect of scale reduction and thermal treatment on multi-material fiber stability. The fiber is inserted in a heating zone for a fixed time interval before both fiber ends are pulled symmetrically in opposite directions [Fig. 1(a)]. The three controllable tapering parameters [inset of Fig. 1(a)] are (1) temperature $T$, which

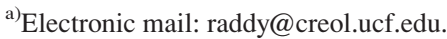

determines the viscosity, (2) tapering distance $\ell$, which determines the size reduction, and (3) tapering speed $v$, which determines the dwelling time in the heating zone. The fiber used in this study consists of a chalcogenide glass $\left(\mathrm{As}_{2} \mathrm{Se}_{3}\right)$ core and a thermoplastic polymer cladding [polyethersulfone (PES), Fig. 1(b)]. A typical taper is shown in Fig. 1(c). The initially continuous core has evolved into a periodic string of droplets in the tapered section [Fig. 1(d)]. This highly robust observation is a manifestation of the PR capillary instability culminating in fiber-core breakup, as we proceed to demonstrate. The full process is shown in Fig. 1(e) with increasing $T$ (at fixed $v$ and $\ell$ ). At lower $T$, the viscosity is large [Fig. 1(f)], and the core remains intact. As $T$ increases, the viscosity drops and surface tension overcomes the inertial viscous force, leading to instability growth until droplets are formed.

We first examine the effect of static fiber heating $(v=0)$ on the core stability. Since the result is "frozen" in the fiber, we can monitor the details of the instability growth. We heat identical fibers for progressively longer intervals, dissolve the cladding using an organic solvent, ${ }^{14}$ and obtain scanning electron microscope (SEM) micrographs of the core [Fig. 2(a)]. Although the PR instability is initiated through thermodynamic fluctuations at the core-cladding interface, ${ }^{12}$ single instability wavelengths are favored by the process and grow without bound resulting in core breakup. ${ }^{16}$ A shallow sinusoidal modulation first appears at the interface [Fig. 2(b)] and the modulation depth increases [Fig. 2(c)] until droplets form [Fig. 2(d)]. A bridge connects the "mother" droplets until pinch-off, at which point the droplets detach leaving a satellite in the middle [Fig. 2(e)]. ${ }^{19}$ The satellite in turn undergoes the same capillary instability, breaking up into sub-satellites at a smaller scale [Fig. 2(f)]. This fractal process continues at progressively smaller scales down to a fourth generation as shown in Fig. 2 (g) (a total of 9 satellite droplets between mother droplets). ${ }^{19}$

We measured the breakup time $\tau_{B}$ (heating time needed until core breakup) in the static case and find that it depends inversely on $T$ [Fig. 3(a)]. To analyze these results quantitatively we adopt the linear stability analysis of Tomotika ${ }^{16}$ which was developed for a stationary viscous incompressible 

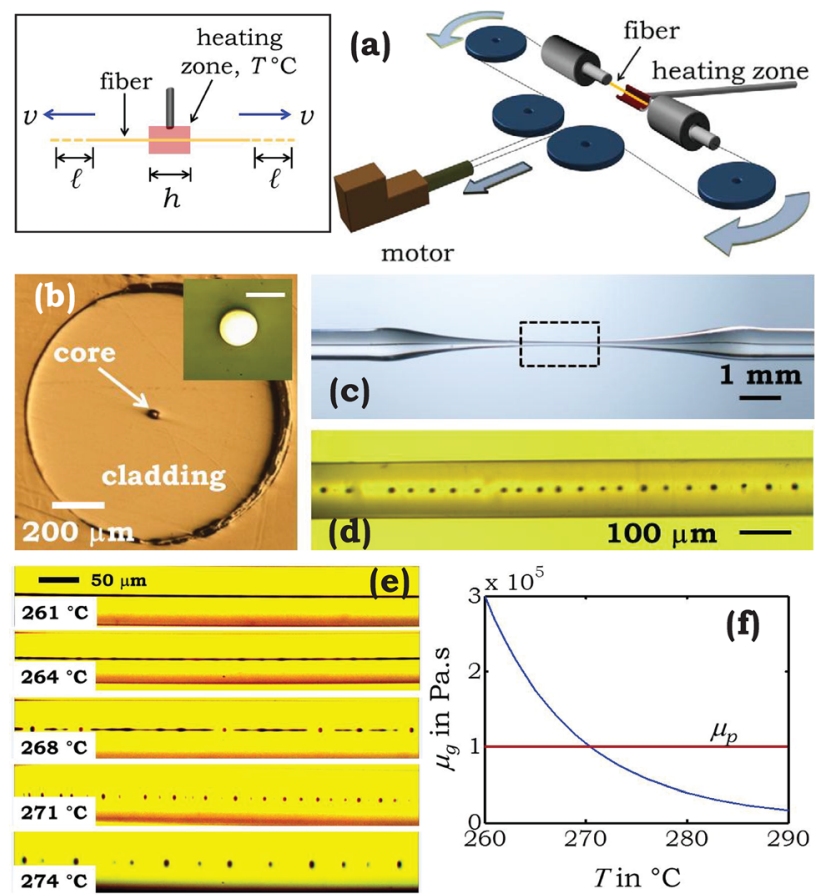

FIG. 1. (Color online) (a) Tapering setup. Inset shows the tapering parameters. (b) Optical micrograph of a fiber cross section. Inset shows a magnified image of the core (scale bar is $20 \mu \mathrm{m}$ ). (c) A typical fiber taper side view. (d) Magnified side view image of the taper center (corresponding to the dotted box in (c)) showing the broken core. (e) Optical micrographs of the fiber tapers side view produced at different $T(v=0.5 \mathrm{~mm} / \mathrm{s}$ and $\ell=15 \mathrm{~mm})$ showing different stages of the core breakup driven by the PR capillary instability. (f) Glass viscosity $\mu_{g}$ as a function of $T$.

fluid thread embedded in an unbound viscous fluid. The glass core viscosity ${ }^{20,21} \mu_{g}$ ranges from $1.75 \times 10^{5} \mathrm{~Pa} . \mathrm{s}$ at $265^{\circ} \mathrm{C}$ to $2.5 \times 10^{4}$ Pa.s at $285^{\circ} \mathrm{C}$ [Fig. 1(f)] and for PES is ${ }^{20} \mu_{p}=10^{5}$ Pa.s (assumed constant at the temperatures of interest). Surface tension is $\gamma=0.1 \mathrm{~N} / \mathrm{m}$ between $\mathrm{As}_{2} \mathrm{Se}_{3}$ and $\mathrm{PES},{ }^{20}$ and $x=k a=\pi D / \lambda$ is a dimensionless parameter, $k=2 \pi / \lambda$, where $\lambda$ is the perturbation wavelength, $a$ is the core radius, and $D$ is the core diameter. In the static case $\tau_{B}(T)=\xi(T) D$ and $P_{B}=\frac{\pi}{x_{m}} D$, where $x_{m}$ is the value of $x$ that minimizes $\tau_{B}$
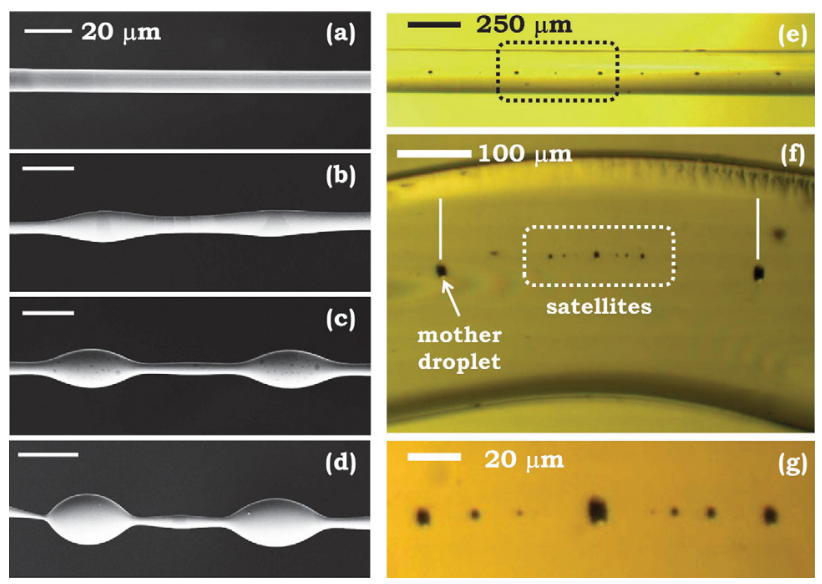

FIG. 2. (Color online) (a)-(d) SEM micrographs of the fiber core at different stages of the PR instability during static heating at $287^{\circ} \mathrm{C}$. Scale bars are all $20 \mu \mathrm{m}$. (e) Optical micrograph of the fiber after core breakup. (f) A single period corresponding to the dotted box in (e). In order to make the satellites visible, the fiber is squeezed between two microscope slides. (g) Magnified image of the satellites and sub-satellites corresponding to the dotted box in (f). for a given wavelength, $P_{B}=\lambda$ is the breakup period, and $\frac{1}{\xi(T)}=\frac{\gamma}{\mu_{p}}\left(1-x^{2}\right) \Phi\left(x_{m}, \frac{\mu_{g}(T)}{\mu_{p}}\right)$, where $\Phi$ is given by Eqs. (39) and (40) in Ref. 16. The calculated instability growth rate for static heating [Fig. 3(b)] is in agreement with the data in Fig. 3(a). Although the Tomotika theory is based on an unbound surrounding fluid, the ratio of cladding to core diameters is sufficiently large here $(\sim 50)$ to justify this approximation.

In order to describe the breakup process during tapering, we adopt a quasi-static model that decouples the instability growth from the tapering dynamics. This may be justified by the difference in time scales of the two processes: the slow breakup and the rapid variation in size during tapering. This model, despite its simplicity, yields results in good agreement with our observations. The taper diameter at time $t$ after start of tapering is $D(t, v)=D_{i} \exp \{-v t / h\}$, where the initial core diameter at $t=0$ is $D_{i}=10 \mu \mathrm{m}$ and $h$ is the length of the heating zone $(\approx 7.5 \mathrm{~mm})$. This formula is derived utilizing geometric considerations and simplifying but reasonable physical assumptions: (1) conservation of volume, (2) a uniform spatial distribution of temperature in the heating zone, and (3) the material exiting the heating zone cools down immediately. If breakup occurs at $t=\tau_{B}$, the core diameter is
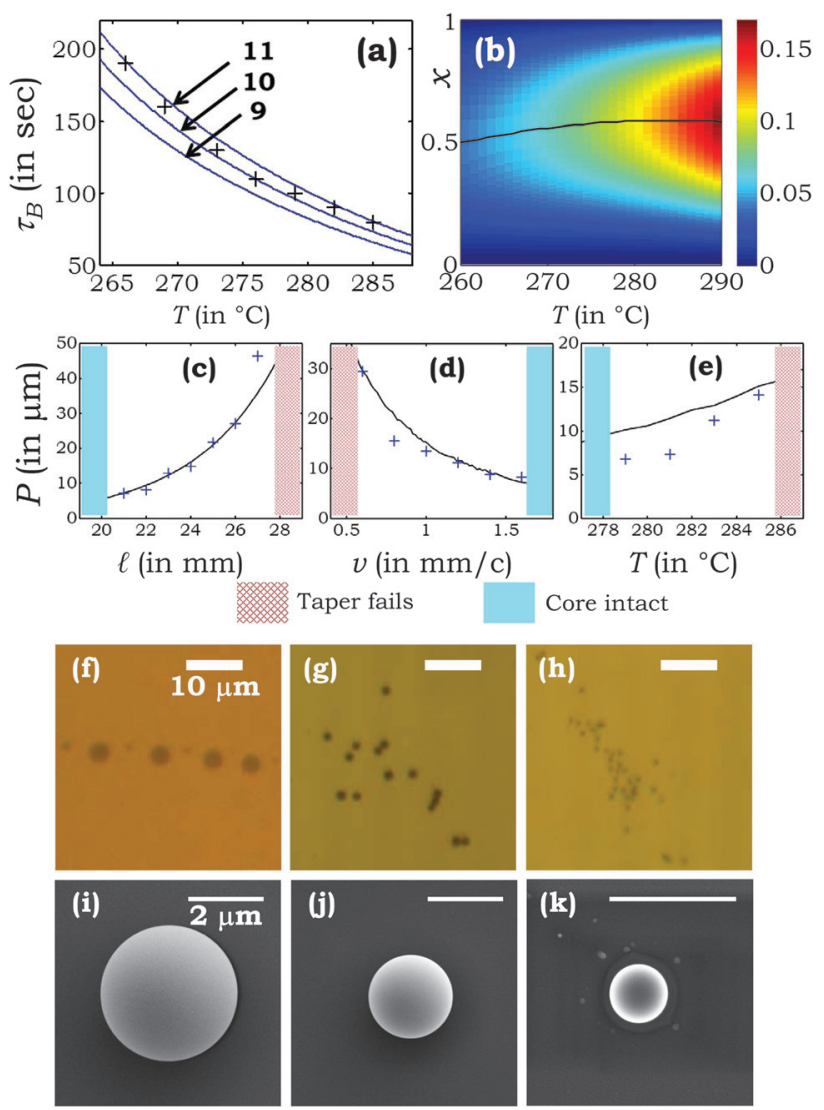

FIG. 3. (Color online) (a) Dependence of the instability growth time $\tau_{B}$ on temperature $T$ during static heating. We calculate and plot $\tau_{B}$ over the same temperature range for $D=9,10$, and $11 \mu \mathrm{m}$. (b) Calculated instability rate $\left(1 / \tau_{B}\right)$ as a function of $T$ and $x$. The black line shows the highest instability rate as a function of temperature, thus identifying $x_{m}$. (c)-(e) Effect of tapering parameters on core breakup period $P_{B}$ : (c) $\ell$, (d) $v$, and (e) $T$. Continuous curves are theoretical fits and "+" are data points. (f)-(h) Optical micrographs of glass particles with prescribed sizes obtained after dissolving the polymer cladding. Scale bars are all $10 \mu \mathrm{m}$. (i)-(k) SEM micrographs of single particles corresponding to (f)-(h), respectively. Scale bars are all $2 \mu \mathrm{m}$. 
$D\left(\tau_{B}, v\right)$ and the period is $P_{B}$. If $v \tau_{B}>l$, the core remains intact. Taylor ${ }^{22}$ observed in an analogous arrangement that an outer viscous fluid delays the onset of the PR instability in an extending inner fluid thread. In light of this, we have found that an empirical correction term $\Delta \tau_{B} \propto 1 / v$ to $\tau_{B}$ yields a good fit to our data within the range of $v$ used in our experiments. The breakup time and period in this case are $\tau_{B}(v, T)=\xi(T) D\left(\tau_{B}, v\right)+\Delta \tau_{B}(v) \quad$ and $\quad P_{B}=\frac{\pi}{x_{m}} D\left(\tau_{B}, v\right)$, respectively. The breakup time $\tau_{B}(v, T)$ now involves the hydrodynamic instability parameters in $\xi(T)$ and the tapering kinematics in $D\left(\tau_{B}, v\right)$. Our model for the excess time $\Delta \tau_{B}$ required for breakup provides an excellent fit to our experimental findings after the proportionality constant (the only fitting parameter in our model) was determined empirically. Subsequent tapering after breakup $\left(\ell>v \tau_{B}\right)$ results in an increase in the inter-particle separation period according to $P(\ell, v, T)=P_{B} \exp \left\{2\left(\ell-v \tau_{B}\right) / h\right\}$. We thus have a predictive theory for the dependence of the final inter-particle separation $P$ on the tapering parameters.

When we increase $\ell\left[v=1 \mathrm{~mm} / \mathrm{s}, T=285^{\circ} \mathrm{C}\right.$; Fig. 3(c) $]$, the core remains intact until $\ell=21 \mathrm{~mm}$, after which it breaks up, and further elongation increases the droplet separation. Next consider the effect of $v\left[\ell=23 \mathrm{~mm}, T=285^{\circ} \mathrm{C}\right.$; Fig. 3(d)]. At high $v$, the dwelling time in the heating zone may be less than $\tau_{B}$ and the core remains intact. At lower $v$, the fiber dwells longer, thus having the opportunity to reach $\tau_{B}$ and hence breaks up. Consequently, tapering at high speeds allows us to reach smaller-diameter cores before breakup. Finally, consider the effect of $T[v=1 \mathrm{~mm} / \mathrm{s}, \ell=23 \mathrm{~mm}$; Fig. 3(e)]. At low $T$, the viscosity of the core is relatively large and the core remains intact. As $T$ increases, the PR instability grows until droplets are formed and $P$ increases with $T$. The quality of the fit in Fig. 3(e) is less than in Figs. 3(c) and 3(d) which show the effect of kinematic parameters $v$ and $\ell$. This is most likely due to the approximations used in the temperature dependence of $\mu_{p}$ and $\mu_{g}{ }^{20,21}$ Our linear model does not consider the strongly nonlinear processes that take place during pinch-off and droplet breakup. Nevertheless, since these later stages occur at much faster time scales than that of the initial instability development, it is expected that our model will still provide a reasonable prediction of $P$.

The process described here offers an unconventional topdown approach to fabricating solid micro-structures with tunable size. Particles of prescribed size are produced in two steps: the fiber is tapered at high speed $\left(v \tau_{B}>l\right)$ to reach an intactcore-diameter $D$, after which it is heated to induce breakup into spheres with diameter $D_{s}=\sqrt[3]{\frac{3 \pi}{2 x_{m}}} D$. In general if the core diameter is $D$ at breakup, then the particle diameter is $\sim 2 D$. Temperature and tapering rate may be used as parameters to control the onset of the Plateau-Rayleigh instability, thus indirectly the resulting particle diameter. To demonstrate size control, we produce particles at three different sizes: 5,2 , and $1 \mu \mathrm{m}$ [Figs. 3(f)-3(k)]. Using dynamic light scattering (DLS) measurements we have established that the standard deviation of the particle size dispersion is typically $\sim 10 \%$ of the mean particle size. Furthermore, this approach is amenable to a wide range of materials that may be included in multi- material fibers ${ }^{9-11}$ where the instability dynamics are dominated by viscosity and surface tension. Examples of such materials include soft glasses and thermoplastic polymers. ${ }^{10}$ Moreover, since the process starts from a macroscopic preform, it may be possible to obtain particles of complex structure by constructing the preform with the prescribed structure.

In summary, we have reported the observation of the PR capillary instability in multi-material optical fiber cores during tapering, leading to breakup of the core into spherical droplets. A theoretical model was developed that determines the effect of tapering parameters on the core stability. This setting offers a new playground for micro-scale fluid dynamics extending it into new geometries and materials. The physical process described here is an unconventional top-down approach to the fabrication of micro-structures from a variety of materials. Using this approach we have demonstrated control over the particle diameter in the $1-20 \mu \mathrm{m}$ range. It seems possible that this technique may be extended to the fabrication of both larger particles and smaller nanoparticles from a variety of materials that can be processed by thermal drawing. This surface-tension-driven instability results in extremely smooth particle surfaces, making them potentially useful in photonics applications as optical micro-resonators.

We gratefully acknowledge support by CREOL, The College of Optics \& Photonics (UCF), and the NSF (ECCS1002295). We thank K. C. Richardson, N. Carlie, and Z. Ruff for glass preparation, and S. G. Johnson for useful discussions. We thank Y. Fink, M. Z. Bazant, M. J. Soileau, and B. E. A. Saleh for their encouragement and support.

\footnotetext{
${ }^{1}$ J. A. F. Plateau, Acad. Sci. Brux. Mem. 16, 3 (1843).

${ }^{2}$ L. Rayleigh, Proc. R. Soc. 29, 71 (1879).

${ }^{3}$ L. Rayleigh, Philos. Mag. 34, 145 (1892).

${ }^{4}$ J. Eggers and E. Villermaux, Rep. Prog. Phys. 71, 036601 (2008).

${ }^{5}$ S. Chandrasekhar, Hydrodynamic and Hydromagnetic Stability (Clarenden, Oxford, 1961).

${ }^{6}$ J. Eggers, Phys. Rev. Lett. 71, 3458 (1993).

${ }^{7}$ P. P. Bhat, S. Appathurai, M. T. Harris, M. Pasquali, G. H. McKinley, and O. A. Basaran, Nat. Phys. 6, 625 (2010).

${ }^{8} \mathrm{P}$. Russell, Science 299, 358 (2003).

${ }^{9}$ A. F. Abouraddy, O. Shapira, M. Bayindir, J. Arnold, F. Sorin, D. S. Hinczewski, J. D. Joannopoulos, and Y. Fink, Nature Mater. 5, 532 (2006).

${ }^{10}$ A. F. Abouraddy, M. Bayindir, G. Benoit, S. D. Hart, K. Kuriki, N. Orf, O. Shapira, F. Sorin, B. Temelkuran, and Y. Fink, Nature Mater. 6, 336 (2007).

${ }^{11}$ S. Egusa, Z. Wang, N. Chocat, Z. M. Ruff, A. M. Stolyarov, D. Shemuly, F. Sorin, P. T. Rakich, J. D. Joannopoulos, and Y. Fink, Nature Mater. 9, 643 (2010).

${ }^{12}$ P. J. Roberts, F. Couny, H. Sabert, B. J. Mangan, D. P. Williams, L. Farr, M. W. Mason, A. Tomlinson, T. A. Birks, J. C. Knight, and P. St. J. Russell, Opt. Express 13, 236 (2005).

${ }^{13}$ M.-C. Phan-Huy, J.-M. Moison, J. A. Levenson, S. Richard, G. Mélin, M. Douay, and Y. Quiquempois, J. Lightwave Technol. 27, 1597 (2009).

${ }^{14}$ D. S. Deng, N. D. Orf, A. F. Abouraddy, A. M. Stolyarov, J. D. Joannopoulos, H. A. Stone, and Y. Fink, Nano Lett. 8, 4265 (2008).

${ }^{15}$ D. S. Deng, N. D. Orf, S. Danto, A. F. Abouraddy, J. D. Joannopoulos, and Y. Fink, Appl. Phys. Lett. 96, 023102 (2010).

${ }^{16}$ S. Tomotika, Proc. R. Soc. London, Ser. A 150, 322 (1935).

${ }^{17}$ T. A. Birks and Y. W. Li, J. Lightwave Technol. 10, 432 (1992).

${ }^{18}$ L. Tong, R. R. Gattass, J. B. Ashcom, S. He, J. Lou, M. Shen, I. Maxwell, and E. Mazur, Nature 426, 816 (2003).

${ }^{19}$ M. Tjahjadi, H. A. Stone, and J. M. Ottino, J. Fluid Mech. 243, 297 (1992).

${ }^{20}$ S. D. Hart, Ph.D. dissertation, MIT, 2004.

${ }^{21}$ A. Tverjanovich, Glass Phys. Chem. 29, 532 (2003).

${ }^{22}$ G. I. Taylor, Proc. R. Soc. London, Ser. A 146, 501 (1934).
} 